\title{
Image Processing of Optical Vortex and Determination of OAM
}

\author{
Makbule GÖK ${ }^{1 *}$, Koray KÖKSAL ${ }^{2}$ \\ 1 Bitlis Eren Üniversitesi, Lisansüstü Eğitim Enstitüsü, Bitlis, Türkiye \\ 2 Bitlis Eren Üniversitesi, Fen-Edebiyat Fakültesi, Bitlis, Türkiye \\ (ORCID: 0000-0003-3452-0533) (ORCID: 0000-0001-8331-9380)
}

\begin{abstract}
This study aims to analyze the transverse spatial profile of the intensity of optical vortex patterns which have been produced by using fork hologram and spatial light modulator. The properties of intensity distribution has been investigated by using image processing technique. The results will be very useful to understand the change of the intensity profile and the value of orbital angular momentum of optical vortex patterns which are obtained experimentally.
\end{abstract}

Keywords: Image processing, Orbital angular momentum, Twisted light

\section{Optik Vorteks için Görüntü İşleme ve OAM Tayini}

\section{$\ddot{O} \mathbf{z}$}

$\mathrm{Bu}$ çalışma, optik girdap desenlerinin yoğunluğunun enine uzaysal profilini analiz etmeyi amaçlamaktadır. Çatal hologram ve uzaysal sşık modülatörü kullanılarak üretilmiştir. Yoğunluk dağılımının özellikleri görüntü işleme tekniği kullanılarak incelenmiştir. Sonuçlar deneysel olarak elde edilen optik girdap desenlerinin momentumunu, yoğunluk profilinin değişimini ve yörünge açısalının değerini anlamak için çok faydalı olacaktır.

Anahtar kelimeler: Görüntü işleme, Yörüngesel açısal momentum, Bükümlü ışık

\section{Introduction}

Orbital angular momentum (OAM) carrying light which has been named as twisted light or optical vortex beam receive an increasing attention in the field of singular optics due to the potential applications such those optical tweezers [1,2], quantum cryptography [3], free space communication [4], optical trapping and cooling $[5,6]$. Orbital angular momentum of light is a result of topological charge value in azimuthal term, $e^{i \ell \phi}$ which is providing the helical characteristics of the light. Similarly, as in the case of angular momentum value $\bar{\mp} \hbar$ of spin polarized light, twisted light can take the values of $\mp \ell \hbar$.

For the generation of optical vortex beams, some different experimental techniques have been used. Spiral phase plates [7] and dove prisms [8] are methods to produce the constant topological charge carrying light. Allowing dynamical modulation of amplitude and phase, spatial light modulators [9] can be used to generate the optical vortex patterns with the aid of computer-generated holograms.

The inhomogeneous characteristics of transverse profile of an optical vortex beam leads to the optical force gradient which can be used as a strong tool for manipulating some dielectric microparticles, atoms and molecules [10]. In theoretical point of view, Laguerre-Gaussian beams are well defined optical vortices which are the solutions of paraxial wave equation in cylindrical coordinates $[11,12]$. Laguerre-Gaussian (LG) beams can be created by using fork-hologram techniques [13], but of course there can be some differences between the theoretically and experimentally obtained radial profiles.

The transverse profile of an LG beam reads where $\mathrm{E}_{0}$ is the amplitude of electromagnetic field. $\ell$ is known as the topological charge or orbital angular momentum (OAM) of light which is

*Corresponding author: makbule.elif.gok@gmail.com

Received: 04.06.2021, Accepted: 07.12.2021 
characterizing the helical phase change of the electromagnetic field. $\mathrm{w}(z)$ is the $z$-dependent beam waist and $p$ is the radial node.

$$
\vec{E}=E_{0} \frac{C_{\ell, p}}{\sqrt{1+\frac{z^{2}}{z_{\mathcal{R}}^{2}}}} e^{-\frac{\rho^{2}}{w^{2}(z)}}\left(\frac{\sqrt{2} \rho}{w^{2}(z)}\right)^{|\ell|} L_{p}^{|\ell|}\left(\frac{2 \rho^{2}}{w^{2}(z)}\right) \times e^{-i \omega t} e^{i \ell \phi+i k z-i(2 p+|\ell|+1) \tan ^{-1}\left(z / z_{\mathcal{R}}\right)+i \frac{k \rho^{2} z}{2\left(z^{2}+z_{\mathcal{R}}^{2}\right)}} \hat{x}
$$

As seen in Eq. 1, OAM is well defined and described in the phase term of the expression. But it is well known that the OAM value of experimentally produced LG beam cannot be determined easily. In this study, our main aim is to produce the optical vortices, to get the images of the spots and to perform an image processing of the spots to get the information of OAM value. In our work, we obtain OAM value by using an optical technique and image processing technique.

We claim that any simple information (here total intensity) obtained by image processing can give a valuable and a discrete data which indicates the value of OAM.

\section{Material and Method}

It is well known that any experimentally produced optical vortex beam can be theoretically described by the Eq. 1. As a simple technique, computer-generated holograms are quite effective to produce the optical vortex patterns. Although general trend is to use liquid crystal displays to modulate and tune the intensity profile of transmitted light [14], there is a possibility to use DMD displays due to their advantages of rapid refresh rates and amplitude modulation properties $[15,16]$. In this experiment shown schematically in Fig. 1, we used both different techniques (DMD and LCD displays) to obtain computergenerated holographic masks and introduce an experimental setup.

In fig. 1, SLM refers to the liquid crystal display (LCD) based spatial light modulator (SLM) device. The pixels on LCD-SLM can be tuned by computer and it is possible to design the wavefront of laser beam by using holographic phase or amplitude masks [14].

The analytical expression for computer-generated holographic masks can be described as an interference of two exponential functions such that

$$
x_{T}=\left|x_{1}+x_{2}\right|^{2}
$$

where $X_{1}=e^{i k x}$ and $X_{2}=e^{i \ell \phi}$. Here, $\ell$ is the topological charge. This mask should be illuminated with a gaussian beam to get far-field Fraunhofer diffraction patterns [17].

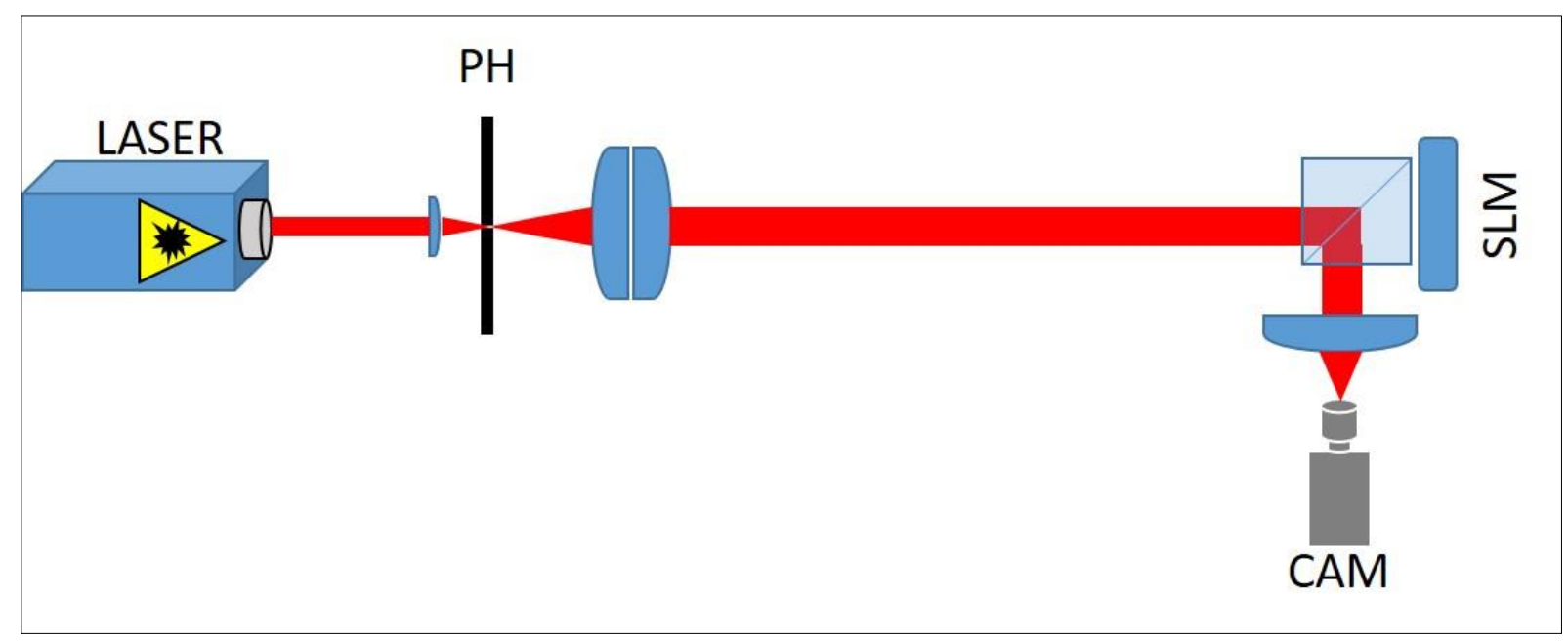


Figure 1. Experimental setup for production and image of the optical vortex patterns. We used $1 \mathrm{~mW} \mathrm{He}-\mathrm{Ne}$ laser with $632.8 \mathrm{~nm}$. PH and CAM indicate the pinhole and CCD camera. Beam splitter and lenses are other elements in the experimental setup

Keeping the wavelength of the laser light constant (here $632.8 \mathrm{~nm}$ ), we change the topological charge $(\ell)$ value of the masks. The obtained radial profiles of the optical vortex patterns are shown in Fig. 2. The analytical expressions and corresponding mask paintings are obtained by using the Mathematica software. The line width between dark fringes should be carefully adjusted to achieve a diffraction.

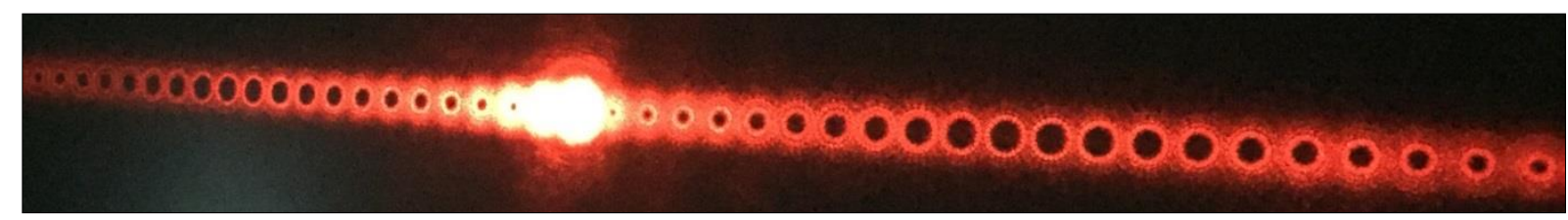

Figure 2. Optical vortex patterns for $\ell=1$ which is obtained by using fork holograms projected on SLM. It is possible to see all patterns with topological charge values from $\ell=-12$ to $\ell=12$

Not only to produce holographic masks, but also to perform image processing, Mathematica is very useful tool to process and analyze any image obtained by optics experiment. Some studies have given effort to analyze the profile of the beam in transverse plane and in propagation directions [18-20]. Here, we concentrate on the relationship between the intensity distribution in radial direction. And we claim that the information about the radial profile of the light can indicate the characteristics of OAM of light.

\section{Results and Discussion}

In this study, we performed to generate the holographic masks by taking the values of topological charge between $-10<\ell<10$. Because of the fork hologram technique, even in the case of $\ell=1$, many optical vortex patterns with different $\ell$ values can be observed in the screen. Fig. 3 shows holographic masks and corresponding first order optical vortex patterns. For every value of $\ell$, the radial profile of the spot in the middle is same with that of original laser spot produced by laser source. The doughnut or ring shape profile can be easily seen on both sides of original spot. The radius of ring is increasing with the value of topological charge. However, it is not possible to observe an obvious difference between leftand right-hand-side spots. One technique to understand the characteristics of the vortex beams can be an interference of two beams. But here our aim is to analyze the beams by using image processing techniques. 


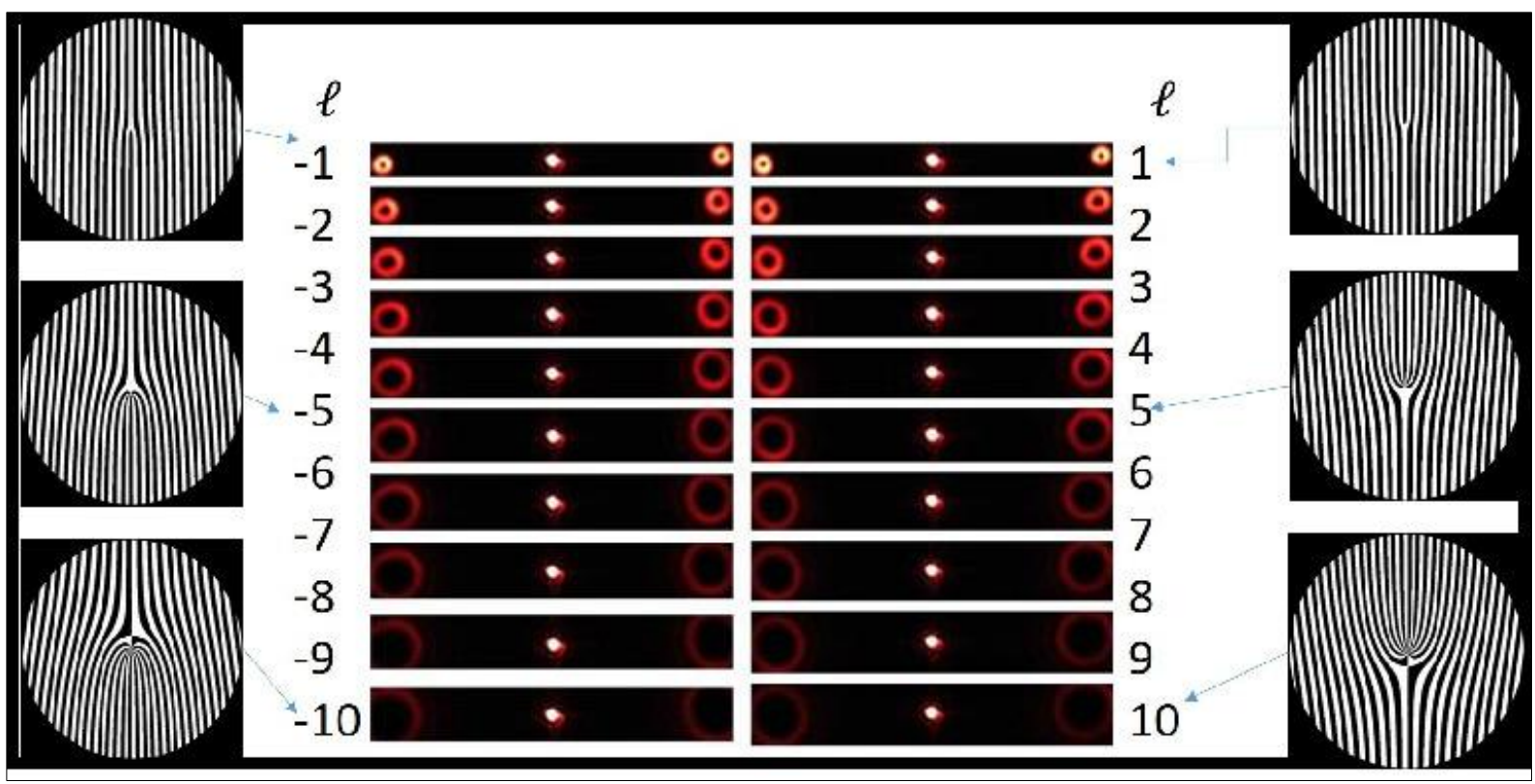

Figure 3. Optical vortex patterns for $\ell=1$ which is obtained by using fork holograms projected on SLM. It is possible to see all patterns with topological charge values from $\ell=-10$ to $\ell=10$

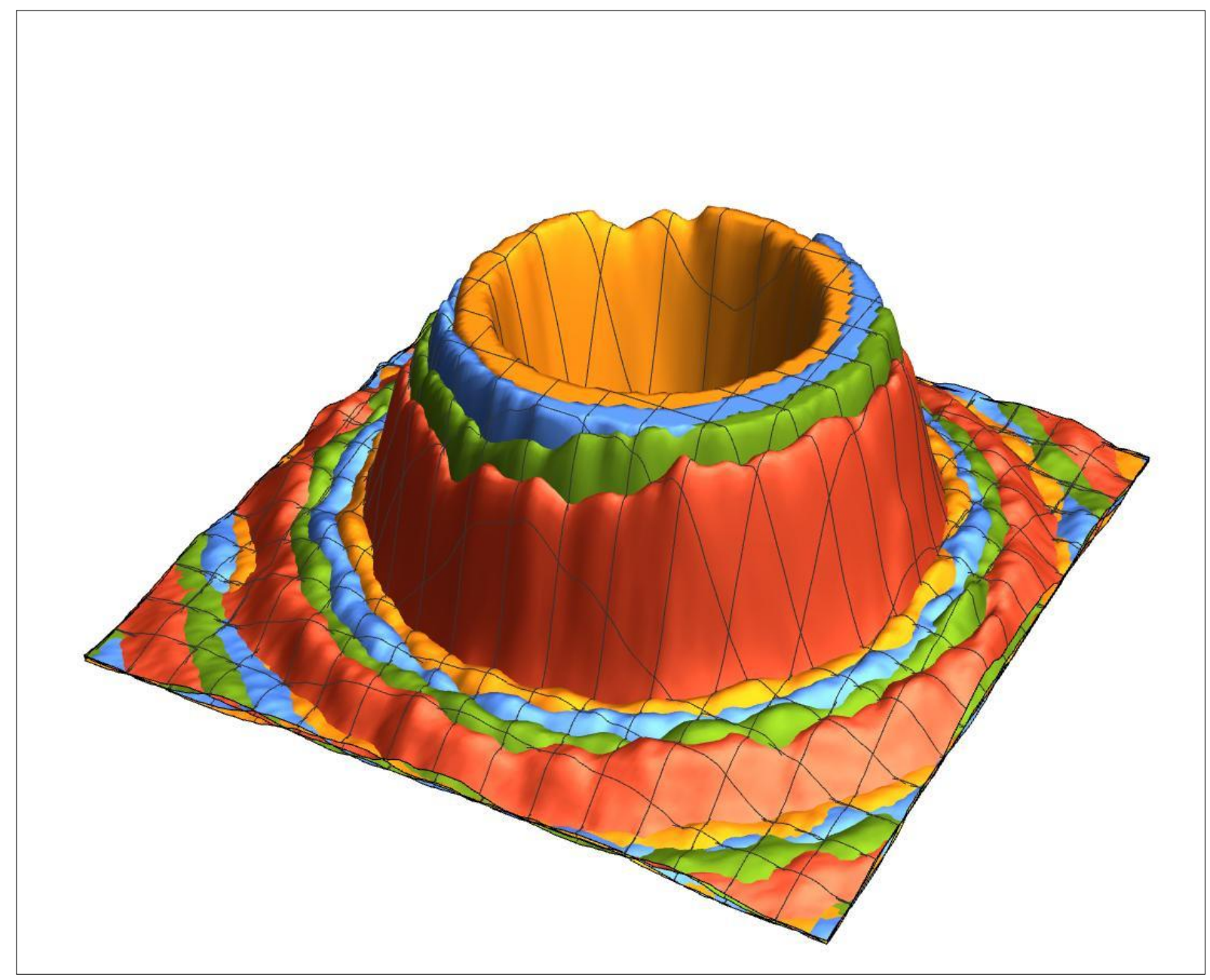

Figure 4. 3D profiles of spots with topological charge values from $\ell=7$ to $\ell=10$ 
2D images of spots to the 3D radial profile of optical vortices by using the image processing technique. One can see the oscillation through radial direction. This oscillation shows that radial node $p$ (see Eq. 1) is different from zero. The term, $\left(\frac{\sqrt{2} \rho}{w^{2}(z)}\right)^{|\ell|}$ in Eq. 1 is indicating an expansion of the dark region of the doughnut shape with the increase in OAM value. The Fig. 4 shows this expansion and the whole properties of the Eq. 1.

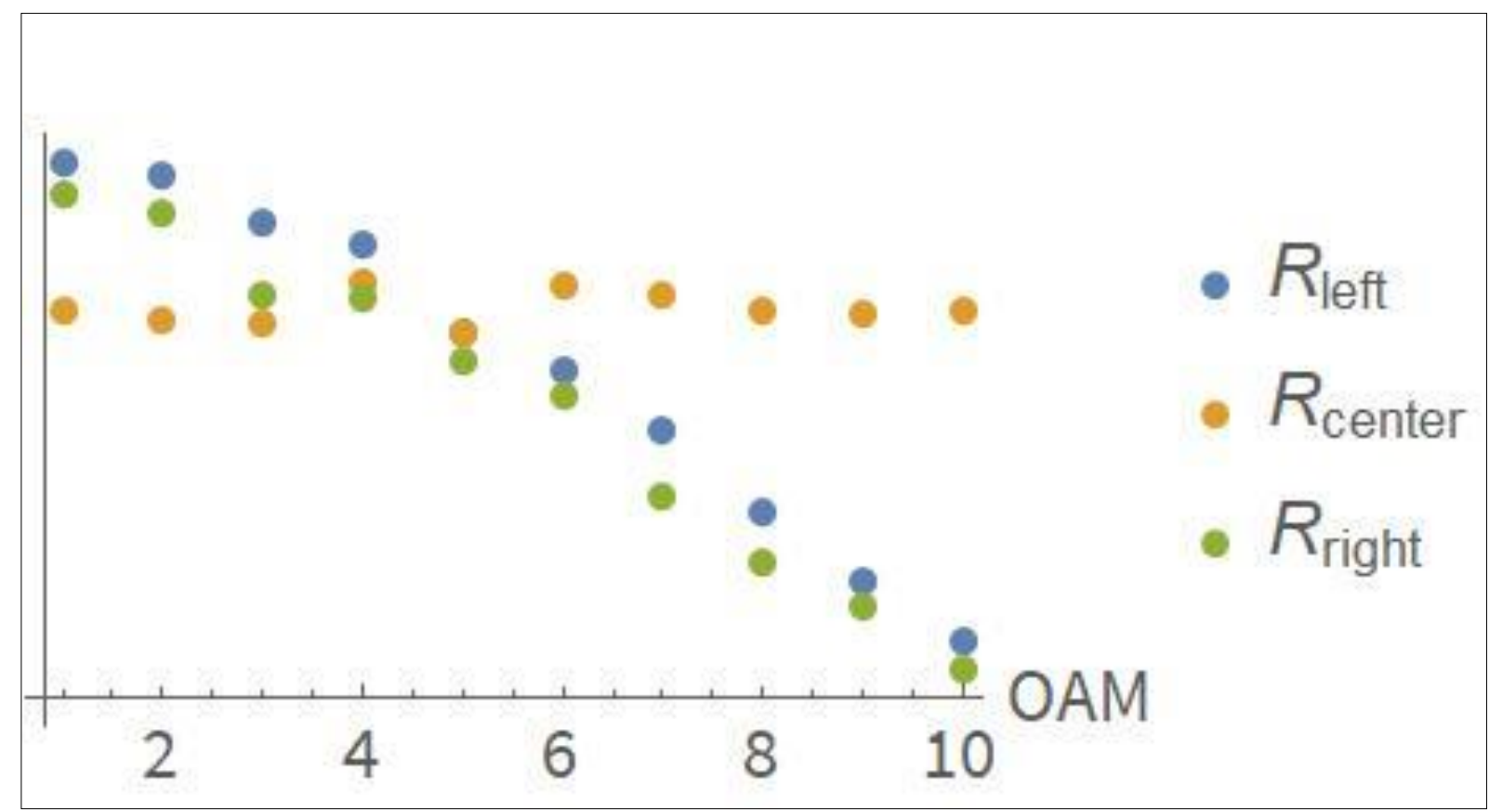

Figure 5. Total intensity of the spots with topological charge values from $\ell=1$ to $\ell=10$

Furthermore, it is also possible to get the total intensity for understanding any change between the spots with different OAM values. In Fig. 5, the bright spots mid of the ring-shaped spots are always indicating $\ell=0$. In Fig. 5, the yellow marks named as " $R_{\text {center }}$ " Show the total intensity values of these bright spots. As seen in the figure, the values of the total intensity of central spots are making an oscillation around a constant value. But the total intensity values of the spots on left $\left(R_{\text {left }}\right)$ and right $\left(R_{\text {right }}\right)$ hand-side of central spots are decreasing with increasing OAM. As we mentioned before, the interference patterns are not limited with these spots. It seems that for lower values of OAM, first two spots are brighter than central spot. For higher values of OAM, central spot is brighter than other ones.

The Fig. 5 which is a result of image processing can be analyzed in another way. By the mean of optical or electronic devices, it is very important to detect OAM or spin angular momentum value of light. In the case of any method using optical techniques. As an example of optical technique to define the OAM value of light, we got the image of the spot which is produced by using two counterpropagating light beams. In Fig. 6, the number of the lobes will give two times OAM value. From this figure, we can directly guess that the OAM is $\ell=5$. However, this technique is not useful in the case of single beam applications.

The result in the Fig. 5 can be used as an example of obtaining the OAM value of light by the mean of electronic tool. Because we got this result by using a digital camera and converted the signals to the numerical values. As seen in the figure, the total intensity value is decreasing with the increasing of OAM. Which means that there is a way to determine the OAM of light with an electronic device. 


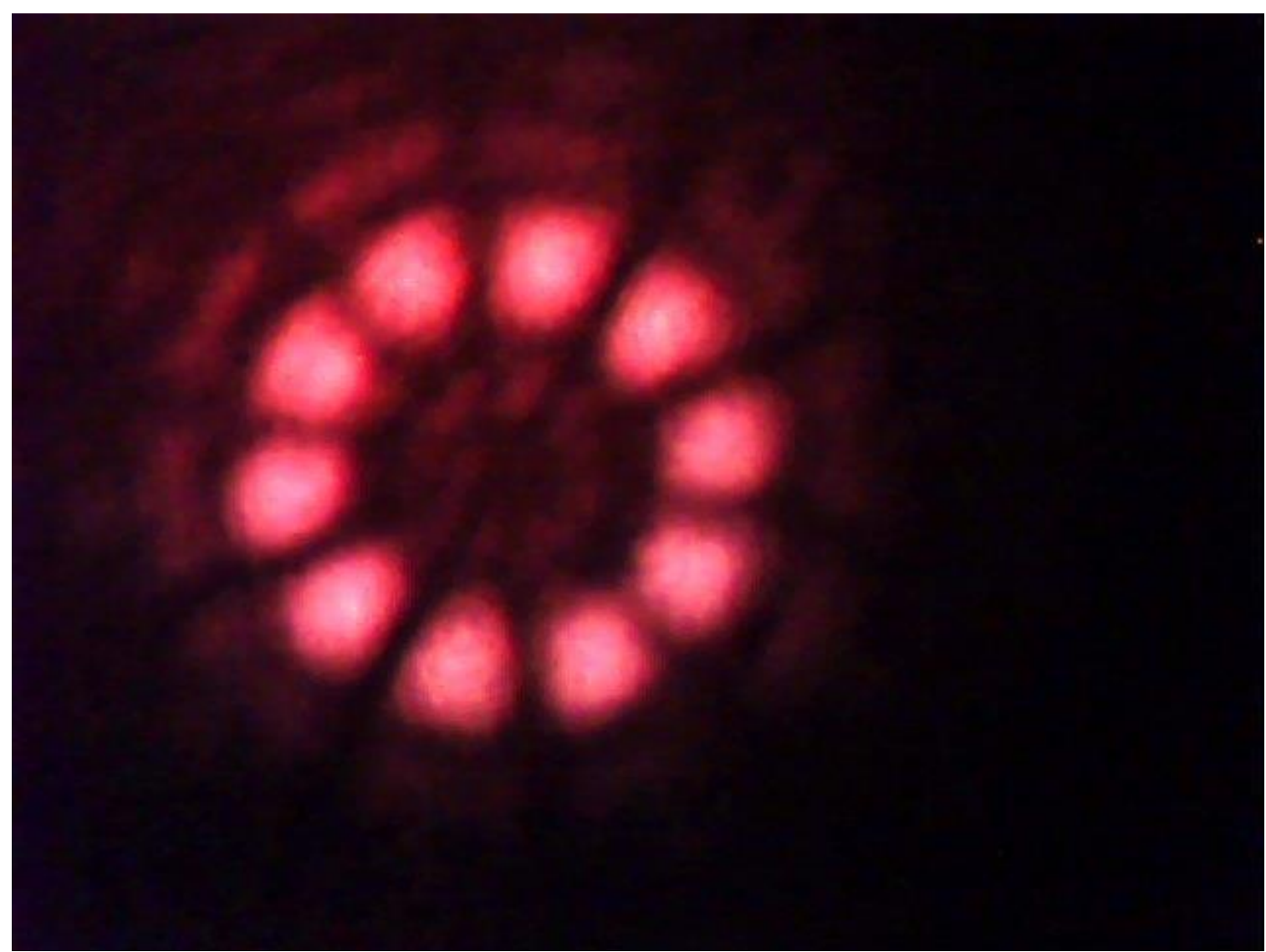

Figure 6. The intensity patterns of two counter-propagating twisted light

\section{Conclusion}

We showed that the value of orbital angular momentum of twisted light obtained by using image processing technique. The total radial intensity of the light is decreasing with the increasing of OAM. Even in the case of lack of a perfect distinction between total intensity values of $-\ell$ and $+\ell$, it is clearly seen that as a reference value, the total intensity of central spot $(\ell=0)$ has a constant value for all values of OAM. The total intensity of right- and left-hand-side spots are varying with respect to the that of central spot. This result indicates that image processing is a useful tool to determine OAM of the light.

\section{Acknowledgment}

This work is supported by Bitlis Eren University Research Projects Office with Grant number BEBAP 2021.10 .

\section{Author's Contributions}

Koray KÖKSAL has planned the experimental setup and written the results. Experiments have been performed by Makbule GÖK.

\section{Statement of Conflicts of Interest}

No potential conflict of interest was reported by the authors.

\section{Statement of Research and Publication Ethics}

The authors declare that this study complies with Research and Publication Ethics. 


\section{References}

[1] Padgett M., Bowman R. 2011. Tweezers with a twist. Nature Photonics, 5: 343-348.

[2] Otte E., Denz C. 2020. Optical trapping gets structure: structured light for advanced optical manipulation. Applied Physics Reviews, 7 (4): 041308.

[3] Sit A., Fickler R., Alsaiari F., Bouchard F., Larocque H., Gregg P., Yan L., Boyd R. W., Ramachandran S., Karimi E. 2018. Quantum cryptography with structured photons through a vortex fiber. Optics Letters, 43: 4108-4111.

[4] Shao W., Huang S., Liu X., and Chen M. 2018. Free-space optical communication with perfect optical vortex beams multiplexing. Optics Communications, 427: 545-550.

[5] Ashkin A. 2006. Optical Trapping and Manipulation of Neutral Particles Using Lasers: A Reprint Volume with Commentaries, World Scientific.

[6] Ng J., Lin Z., Chan C. 2010. Theory of optical trapping by an optical vortex beam. Physical Review Letters, 104: 103601.

[7] Ruffato G., Massari M., Romanato F., 2014. Generation of High-order Laguerre-Gaussian modes by means of spiral phase plates. Optics Letters, 39: 5094-5097.

[8] Gonzalez N., Molina-Terriza G., and Torres J. P. 2006. How a dove prism transforms the orbital angular momentum of a light beam. Optics Express, 14: 9093-9102.

[9] Ostrovsky A. S., Rickenstorff-Parrao C., and Arrizon V. 2013. Generation of the "perfect" optical vortex using a liquid-crystal spatial light modulator. Optics Letters, 38: 534-536.

[10] Ma Y., Rui G., Gu B., Cui Y. 2017. Trapping and manipulation of nanoparticles using multifocal optical vortex metalens. Scientific Reports, 7: 1-9.

[11] Koksal K., Lembessis V. E., Yuan J., Babiker M. 2019. Interference of axially-shifted LaguerreGaussian beams and their interaction with atoms. Journal of Optics, 21, 104002.

[12] Koksal K., Lembessis V. E., Yuan J., Babiker M. 2020. Atoms in axially shifted tightly focused counter-propagating beams: the role of the gouy and curvature phases. JOSA B, 37: 2570-2577.

[13] Bekshaev A., Orlinska O., Vasnetsov M. 2010. Optical vortex generation with a "fork" hologram under conditions of high-angle diffraction. Optics Communications, 283: 2006-2016.

[14] Huang D., Timmers H., Roberts A., Shivaram N., Sandhu A. S. 2012. A low-cost spatial light modulator for use in undergraduate and graduate optics labs. American Journal of Physics, 80: 211-215.

[15] Mirhosseini M., Magana-Loaiza O. S., Chen C., Rodenburg B., Malik M., Boyd R. W. 2013. Rapid generation of light beams carrying orbital angular momentum. Optics Express, 21: 3019630203.

[16] Panarin S., Müller J., Prabhakar S., Fickler R. 2020. Spatial structuring of light for undergraduate laboratory. ArXiv: 2003.12713.

[17] Carpentier A. V., Michinel H., Salgueiro J. R., Olivieri D. 2008. Making optical vortices with computer-generated holograms. American Journal of Physics, 76: 916-921.

[18] Alsultanny Y. A. 2006. Laser beam analysis using image processing. Journal of Computer Science, 2 (1): 109-113.

[19] Bajić J. S., Stupar D. Z., Tomić J., Slankamenać M. P., Joža A., Živanov M. B. 2012. Implementation of The Optical Beam Profiler System Using LabVIEW Software Package and Low-cost Web Camera. In 2012 Proceedings of the 35th International Convention MIPRO, 167172, IEEE.

[20] Hulea M., Ghassemlooy Z., Rajbhandari S. 2013. Evaluation of The Beam Wondering in Free Space Optics by Image Analysis. In Proceedings of the International Conference on Electronics, Computers and Artificial Intelligence-ECAI-2013, 1-4, IEEE. 